\title{
Reconstruction of tau leptons and prospects for SUSY in ATLAS
}

\author{
Carolin Zendler \\ University of Bonn \\ for the ATLAS collaboration
}

SUSY09, Boston 05.06.- | 0.06. 2009

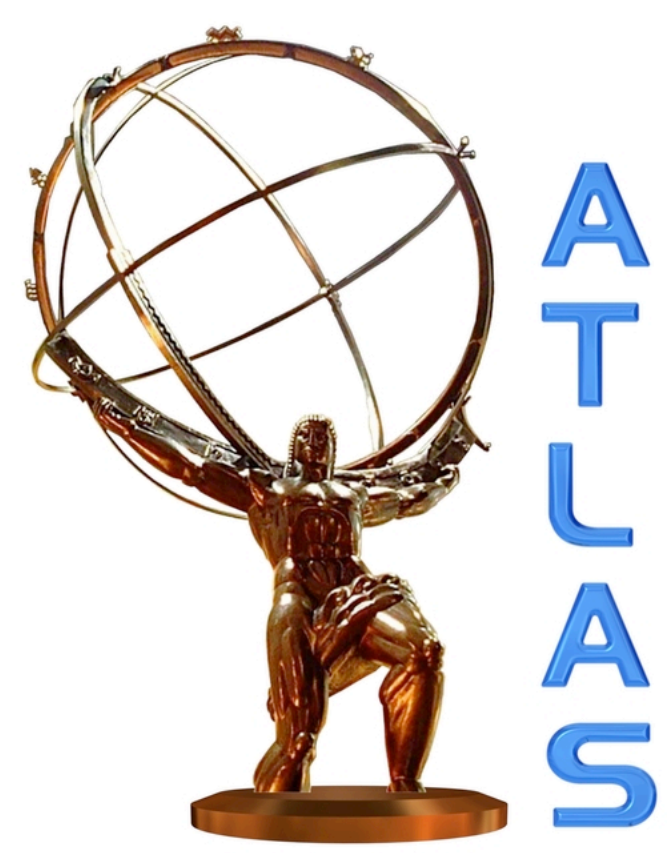




\section{Outline}

I Introduction and Motivation

II Tau reconstruction and identification in ATLAS

III SUSY analyses with tau leptons * Inclusive: discovery prospects * Exclusive: measurement of properties

IV Summary 


\section{Motivation}

\section{Why are taus interesting for SUSY?}

$3^{\text {rd }}$ generation special in SUSY:

mixing of $\tilde{\tau}_{\mathrm{L}}$ and $\tilde{\tau}_{\mathrm{R}}$ to $\tilde{\tau}_{1}, \tilde{\tau}_{2}$

$\rightarrow \widetilde{\tau}_{1}$ and therefore $\tau$ production enhanced

米 $\tilde{\tau}_{1}$ in many models lightest slepton

$\rightarrow \tilde{\chi}_{2}{ }^{0} \rightarrow \tilde{\tau}_{1} \tau$ larger BR than analog e/ $\mu$ decays, may even be only allowed (2body) decay

$\rightarrow$ important discovery channel

tau final states provide unique information not accessible otherwise, e.g. on stau masses

tau decay offers opportunity to measure tau polarization

$\rightarrow$ information about couplings of $\tilde{\chi}_{2}{ }^{0}, \tilde{\chi}_{1}{ }^{0}$ and $\tilde{\tau}_{1}$ 


\section{Introduction: tau leptons}

\section{Tau characteristics:}

* $\mathrm{m}_{\mathrm{T}} \simeq \mathrm{I} .7 \mathrm{GeV}, \mathrm{cT} \simeq 87 \mu \mathrm{m}$

$\rightarrow$ decay within detector,

visible only via decay

products:

$35 \%$ leptonically

$17.8 \% \mathrm{~T} \rightarrow \mathrm{e} \mathrm{v}_{\mathrm{T}} \mathrm{v}_{\mathrm{e}}$

$17.4 \% \mathrm{~T} \rightarrow \mu \nu_{\mathrm{T}} v_{\mu}$

$65 \%$ hadronically

$50.2 \%$ I prong (I charged track)

$15.2 \% 3$ prong

$0.1 \% 5$ prong
Towards tau reconstruction:

e/ $\mu$ from $T$ decay hard to distinguish from prompt $e / \mu$

$\rightarrow$ current algorithms focus on hadronic decays:

I prong (IP):

22.4\% $\mathrm{T} \rightarrow \pi^{ \pm} v_{\mathrm{T}}$

73.5\% $T \rightarrow \pi^{ \pm} v_{T}+n \pi^{0}$

3 prong (3p):

$61.6 \% \mathrm{~T} \rightarrow 3 \pi^{ \pm} v_{\mathrm{T}}$

$33.7 \% \mathrm{~T} \rightarrow \mathbf{3} \pi^{ \pm} v_{\mathrm{T}}+\mathrm{n} \pi^{0}$

$\rightarrow$ tau lepton in detector:

jet of charged

and neutral pions

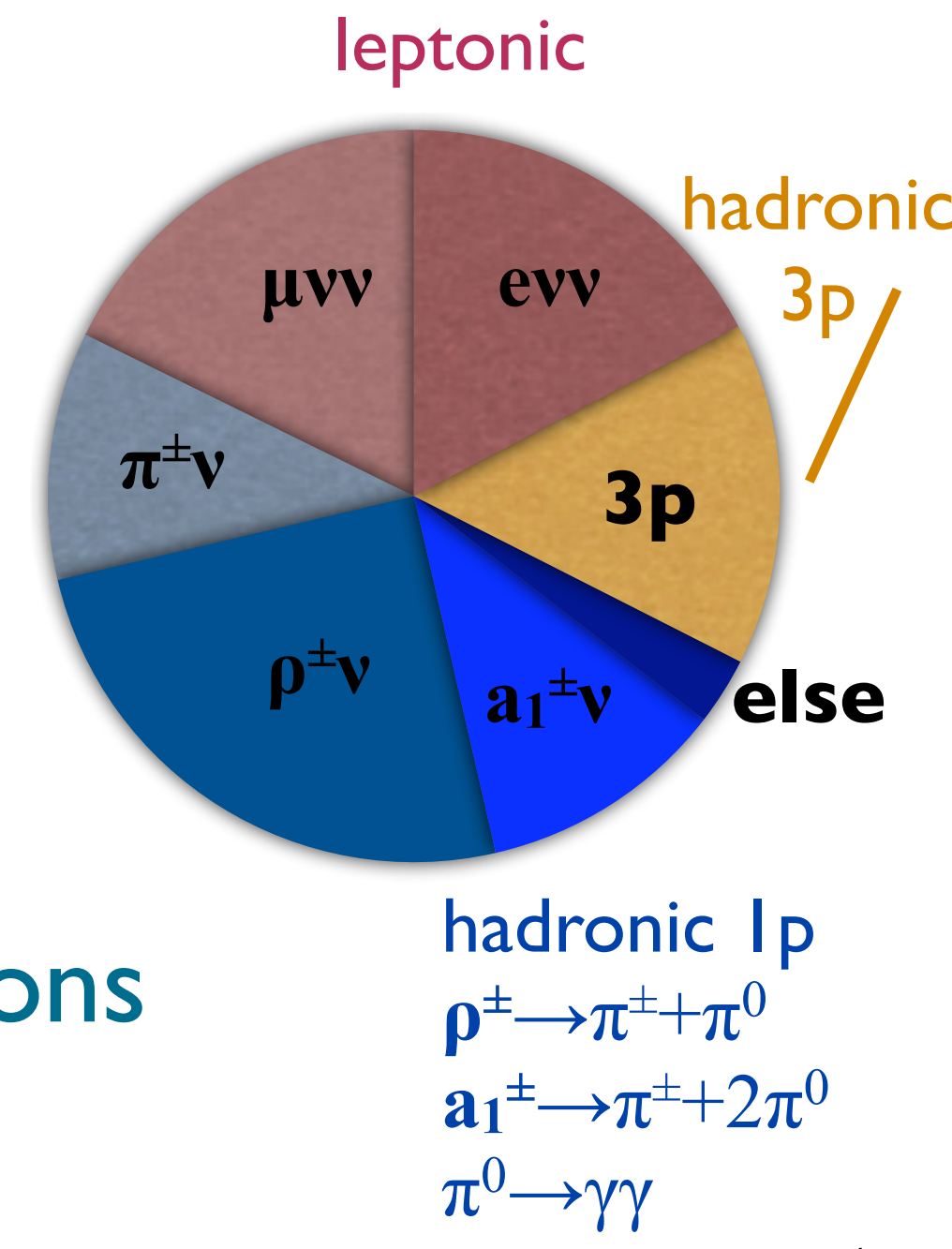




\section{The ATLAS detector}

\section{Ingredients for tau identification:}

\section{Tracking}

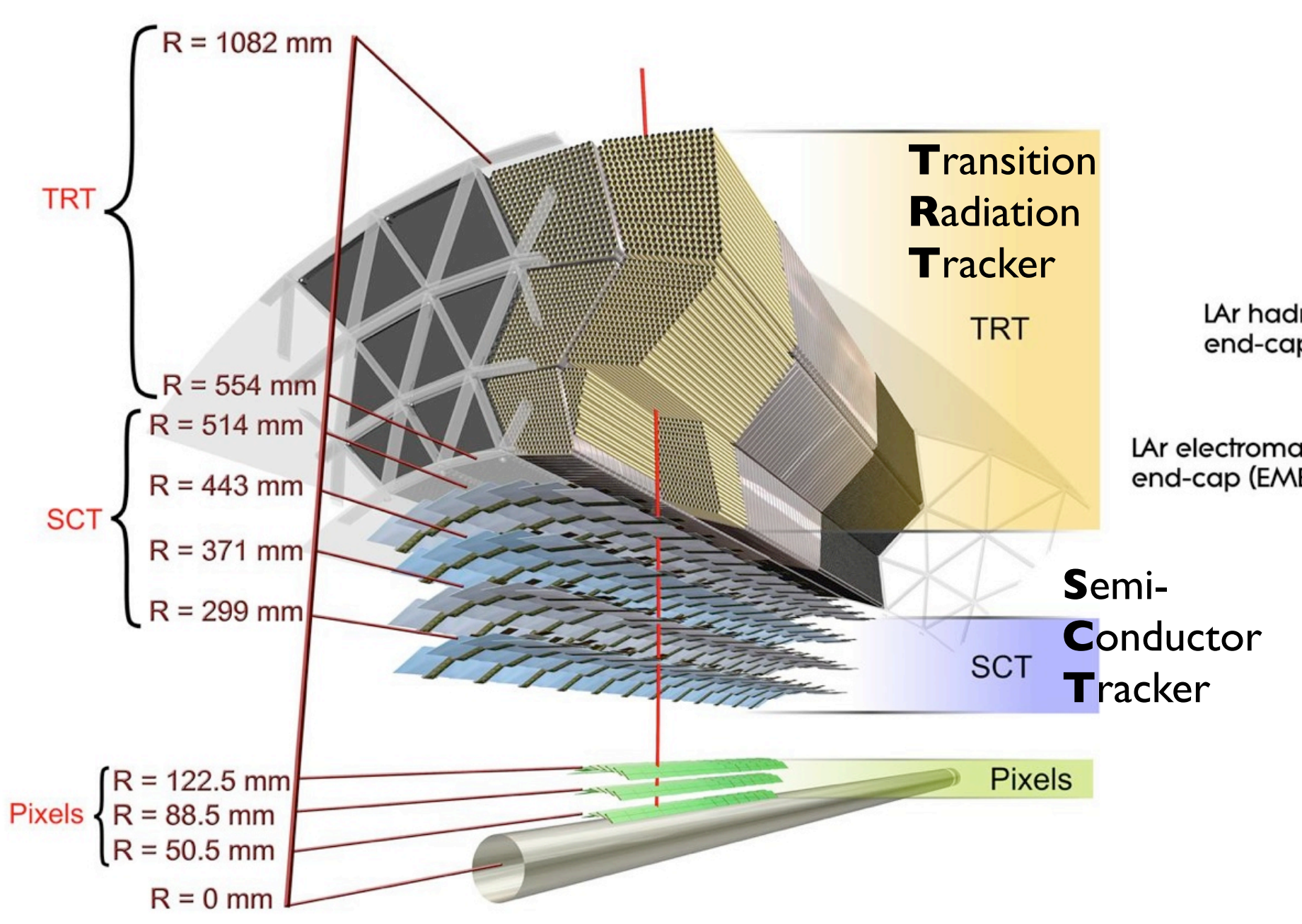

\section{Calorimetry}

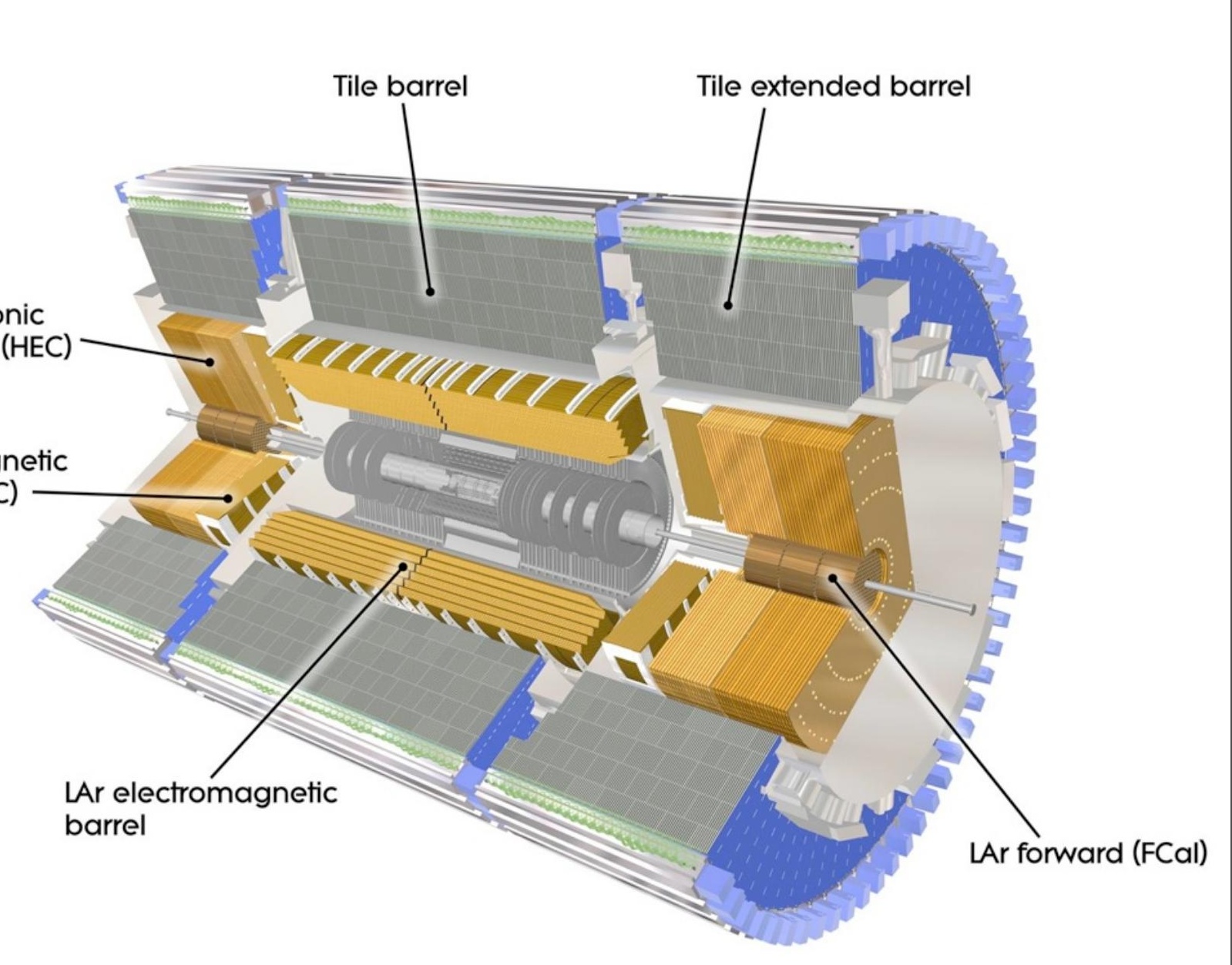

High granularity of sampling calorimeter allows * good shower profile reconstruction

档 reconstruction of $\pi^{0}$ subclusters
䊑 $\mathrm{cT} \simeq 87 \mu \mathrm{m} \rightarrow$ secondary vertex

* Tracking constraint for taus: $|\eta|<2.5$ 


\section{$\tau \mathrm{s}$ from the detector's point of view}

\section{tau}

Two reconstruction algorithms:

* track based: seeded by high quality tracks * calorimeter based: seeded by calorimeter clusters

Main background: QCD jets

Basic distinctive $\tau$ features:

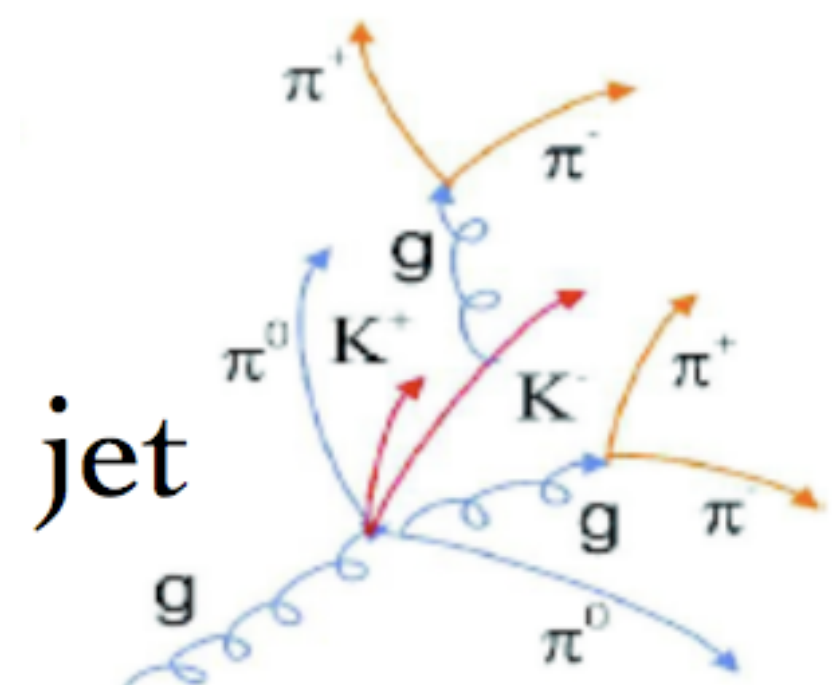
* collimated tracks and energy depositions

* low track multiplicity

* isolation

* impact parameter (I $)$,

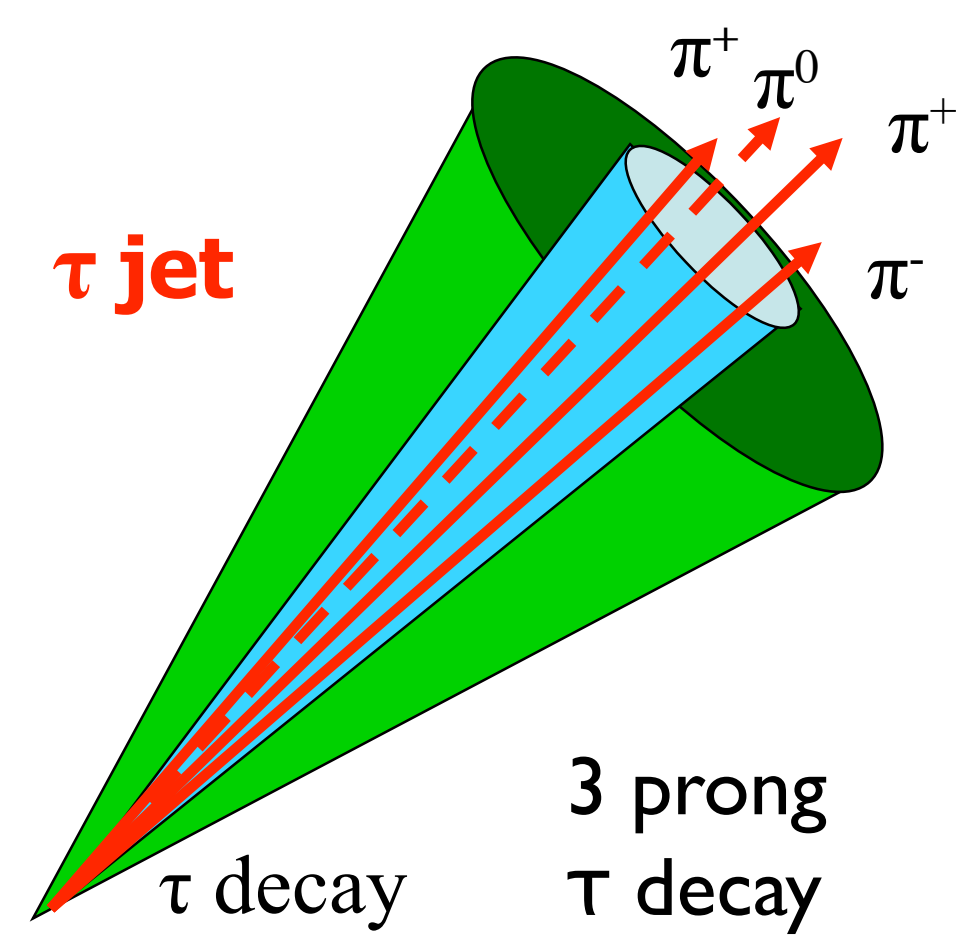

displaced vertex (3p)

* ratio of EM/HAD energy 


\section{Tau reconstruction}

\section{Reconstruction}

use combination of track- and calorimeter-seeded algorithms

* Begin with track based algorithm

* search matching calorimeter seed

$\rightarrow$ no match: track-only candidate $(5 \%)$

$\rightarrow$ match: track+calorimeter seeded candidate (70\%)

* remaining clusters: seeds for calorimeter-only candidates (25\%) 


\section{Tau identification}

\section{Identification}

Many discriminating variables, using

calorimeter and tracking information:

* \#tracks in isolation cone and invariant mass of track system

䊑 shower radius in electromagnetic calorimeter

* \#hits with certain energy deposit in certain calorimeter layer

* $\mathrm{E}_{\mathrm{T}}$ fraction in cone $0 . \mathrm{I}<\Delta \mathrm{R}<0.2$ w.r.t. total energy in cone 0.4 米...

$\rightarrow$ input for different discriminants: cut method, likelihood, neural network, boosted decision trees, PDRS

\section{Identification in early data: "safe variables"}

Reduce complex set of input variables to a few, well understood "safe" variables and use cut-based identification method for early data taking
CERN-OPEN-2008-020

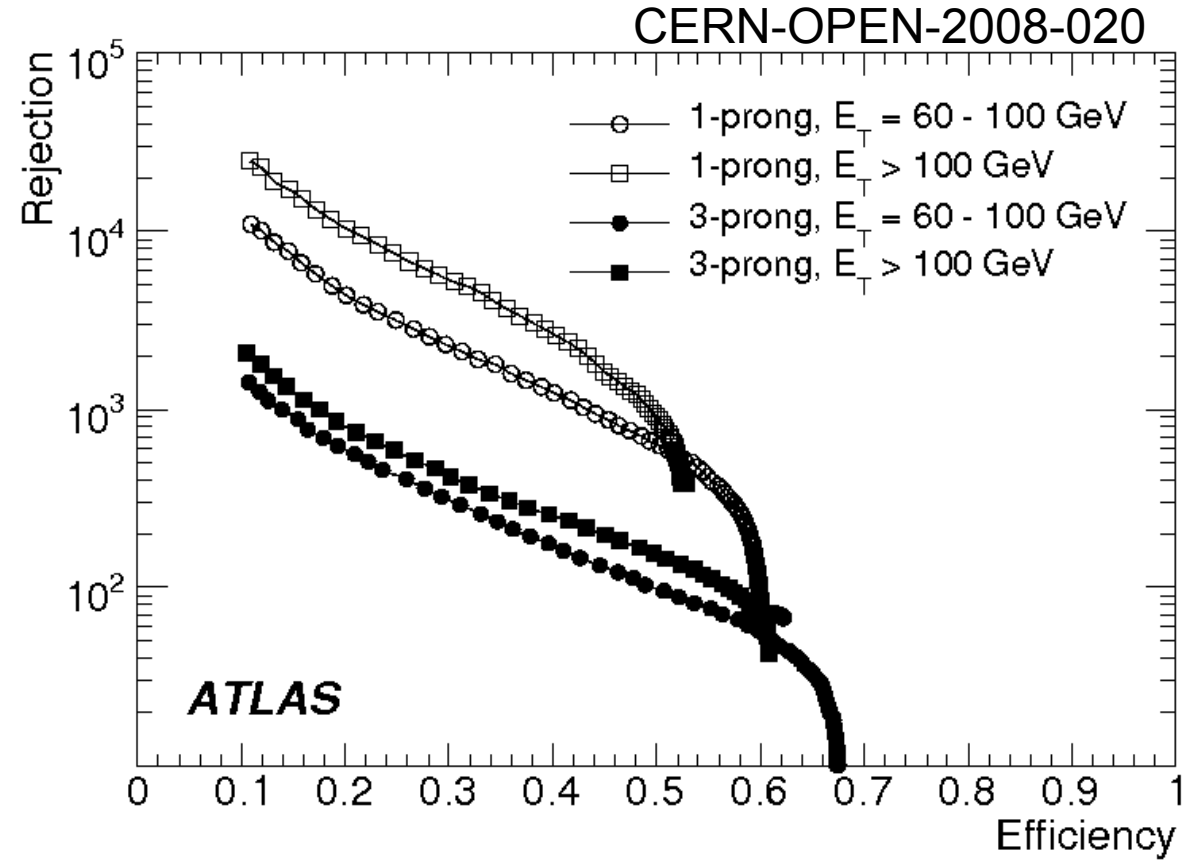

expected performance:

$\uparrow$ calorimeter-based with

likelihood selection

$\downarrow$ track-based, NN selection

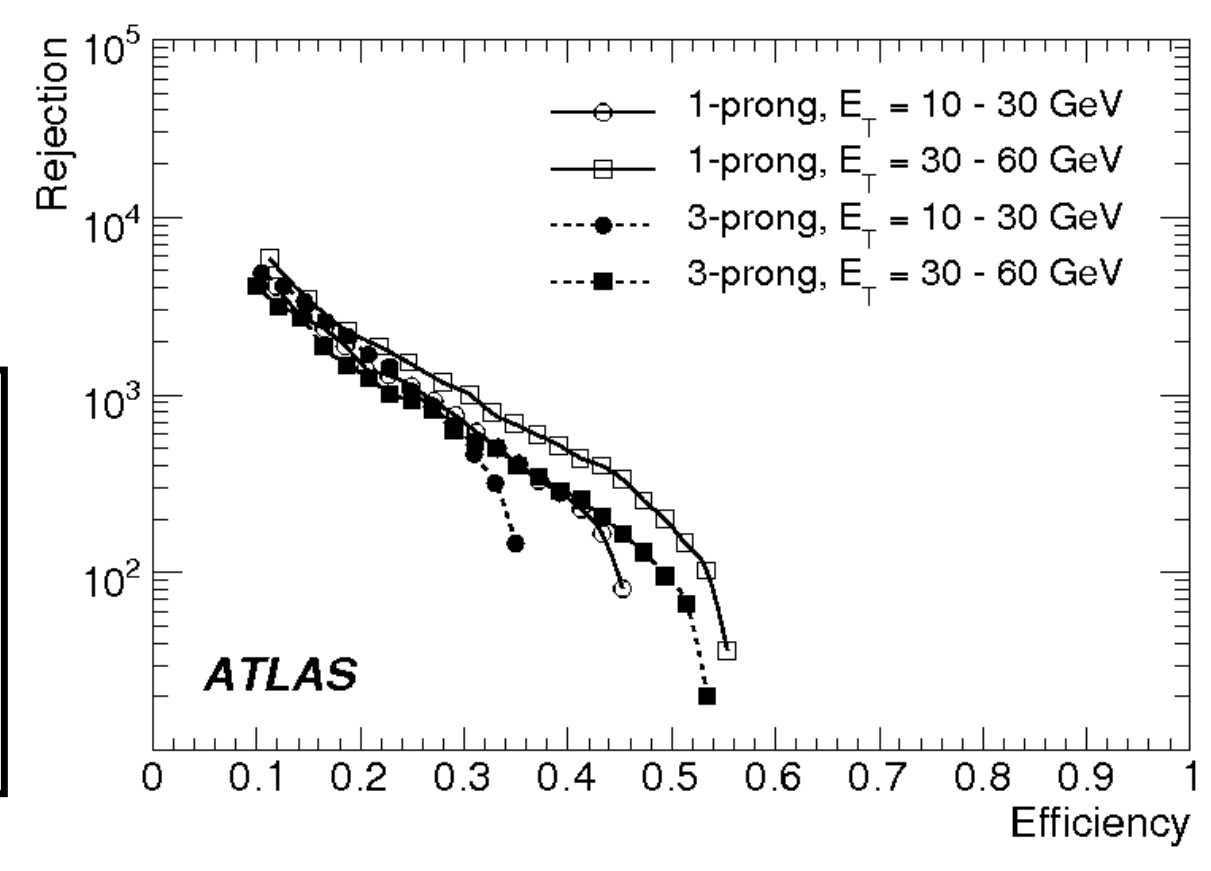




\section{SUSY analyses with tau leptons}

** Inclusive search strategy: cover all possible signatures

$x$ jets $+\mathbf{y}$ leptons/taus $+\mathrm{E}_{\mathrm{T}^{\text {miss }}}$ modes

$\rightarrow$ defined complementary to simplify combination

* development of selection cuts in chosen benchmark points in mSUGRA-like models

* scans of subsets of SUSY parameter space with fast detector simulation

\section{* Exclusive studies: focus on special signatures}

* often very little background

* main goal: measurement of SUSY properties

*⿻丷木 Plots and numbers here: I $\mathrm{fb}^{-1}$ of $14 \mathrm{TeV}$ data

米 $\tau$ identification: track- and calorimeter-based algorithms used separately, safe variables not implemented yet

mSUGRA examples $\rightarrow$ R-parity conservation 


\section{Inclusive searches: tau mode}

\section{Tau mode:}

leptonic $\tau$ decays: part of lepton modes

\section{event selection:}

米 $\geq \mid \tau(\mathrm{pT}>40 \mathrm{GeV},|\eta|<2.5$, calorimeter-based)

$\rightarrow$ efficiency $\approx 50 \%$, purity $\approx 80 \%$ (SU3)

米 no isolated lepton

* 4 jets: $\mathrm{pT}^{>}>50 \mathrm{GeV}$, leading jet: $\mathrm{pT}>100 \mathrm{GeV}$

* $\mathrm{E}_{\mathrm{T}}{ }^{\text {miss }}>100 \mathrm{GeV}, \mathrm{E}_{\mathrm{T}}{ }^{\text {miss }}>0.2 * \mathrm{Meff}$

$\left.\left(\text { Meff }=E_{T}{ }^{m i s}+\sum_{i=1}{ }^{4} \mathrm{PT}^{\text {jet } \mathrm{i}}+\sum_{\mathrm{i}=1} \mathrm{PT}^{\text {lepton }}\right)^{2}\right)$

类 $\Delta \Phi\left(\right.$ jet $\left._{1,2,3}, \mathrm{E}_{\mathrm{T}}{ }^{\text {miss }}\right)>0.2$

* transverse mass of hardest $\tau$ (vis. momentum) and $E_{T}$ miss: $M_{T}>100 \mathrm{GeV}$

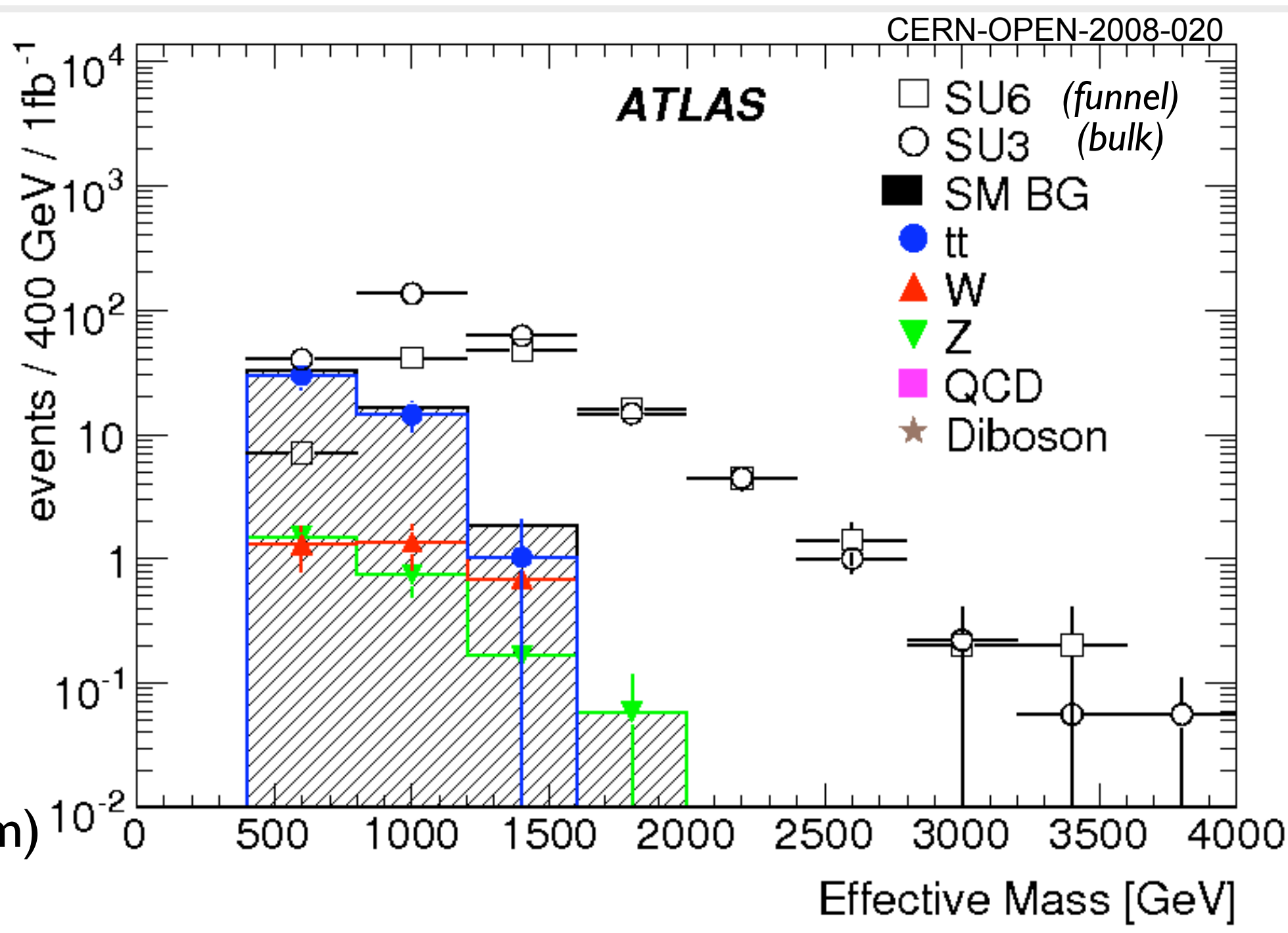

Trigger: $97-100 \%$ efficiency expected when triggering on I jet ( $\left.\mathrm{PT}_{\mathrm{T}}>70 \mathrm{GeV}\right)$ plus $\mathrm{E}_{\mathrm{T}}{ }^{\text {miss }}(>70 \mathrm{GeV})$ (trigger rate: $20 \mathrm{~Hz}\left(\right.$ for $\left.2 \times 10^{33} \mathrm{~cm}^{-2} \mathrm{~s}^{-1}\right)$ )

$\rightarrow$ Expected events at $\mathrm{Ifb}^{-1}$ after tau mode selection cuts: (S: signal, B: background)

\begin{tabular}{|c|c|c|c|}
\hline & S & B & $S / \sqrt{B}$ \\
\hline SU3 & 259 & 51 & 36.3 \\
\hline SU6 & 119 & 51 & 16.7 \\
\hline
\end{tabular}




\section{Exclusive studies: measurements}

\section{Exclusive studies:}

**For SUSY discovery:

show it is SUSY

$\rightarrow$ need to measure properties

米 no mass peaks because of missing LSPs

$\rightarrow$ kinematic edges

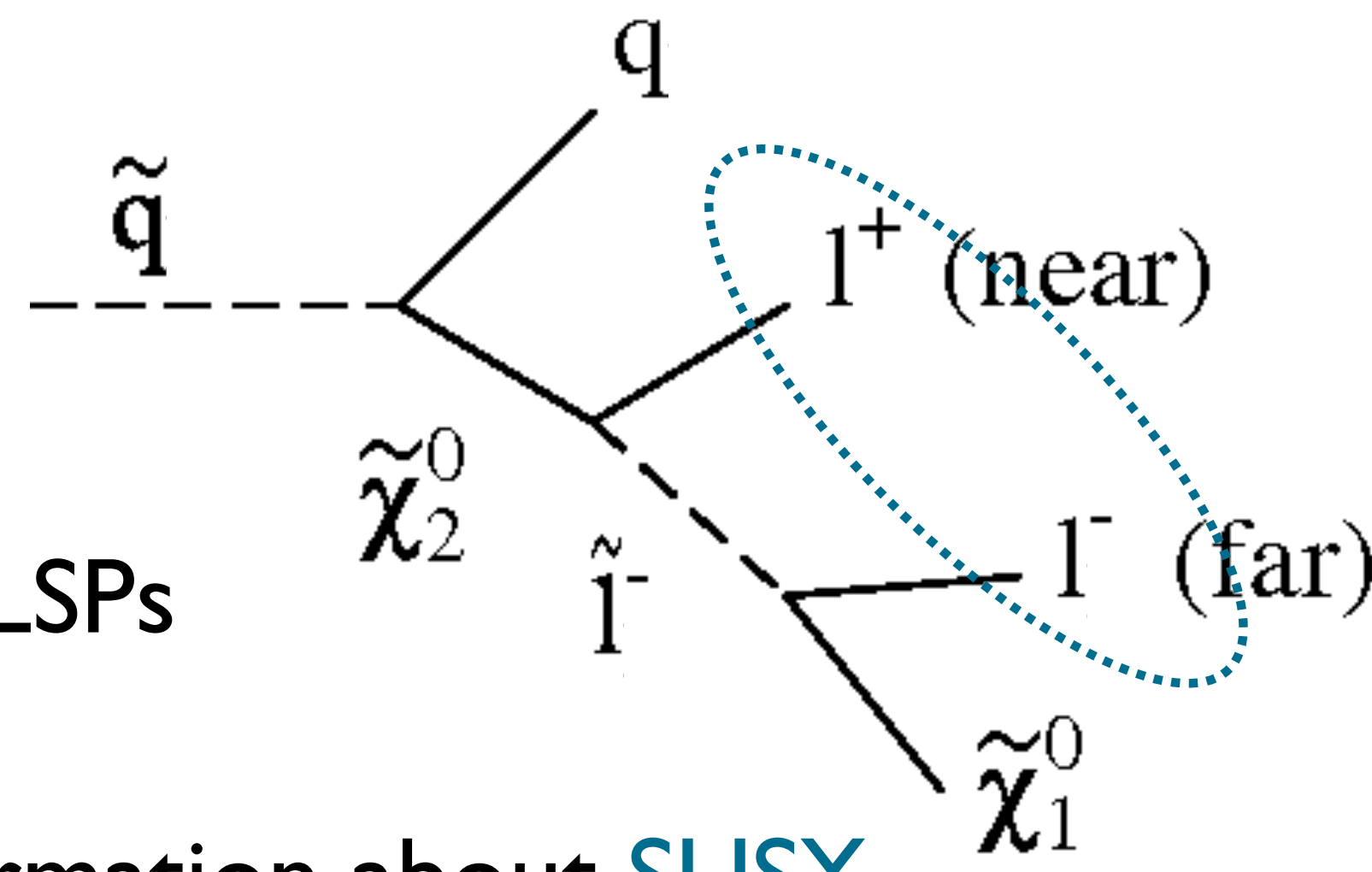

* dilepton mass spectrum holds information about SUSY masses involved in the decay chain: $\tilde{\boldsymbol{\chi}}_{1}{ }^{0}, \tilde{\mathbf{e}} / \tilde{\boldsymbol{\mu}} / \tilde{\mathbf{T}}, \tilde{\boldsymbol{\chi}}_{2}{ }^{0}$

$$
m_{T \tau}^{\max }=\sqrt{\frac{\left(m\left(\tilde{X}_{2}^{0}\right)^{2}-m\left(\tilde{\tau}_{1}\right)^{2}\right) \cdot\left(m\left(\tilde{\tau}_{1}\right)^{2}-m\left(\tilde{X}_{1}^{0}\right)^{2}\right)}{\left(m\left(\tilde{\tau}_{1}\right)^{2}\right)}}
$$

* shape of ditau mass spectrum also holds information about stau mixing angle

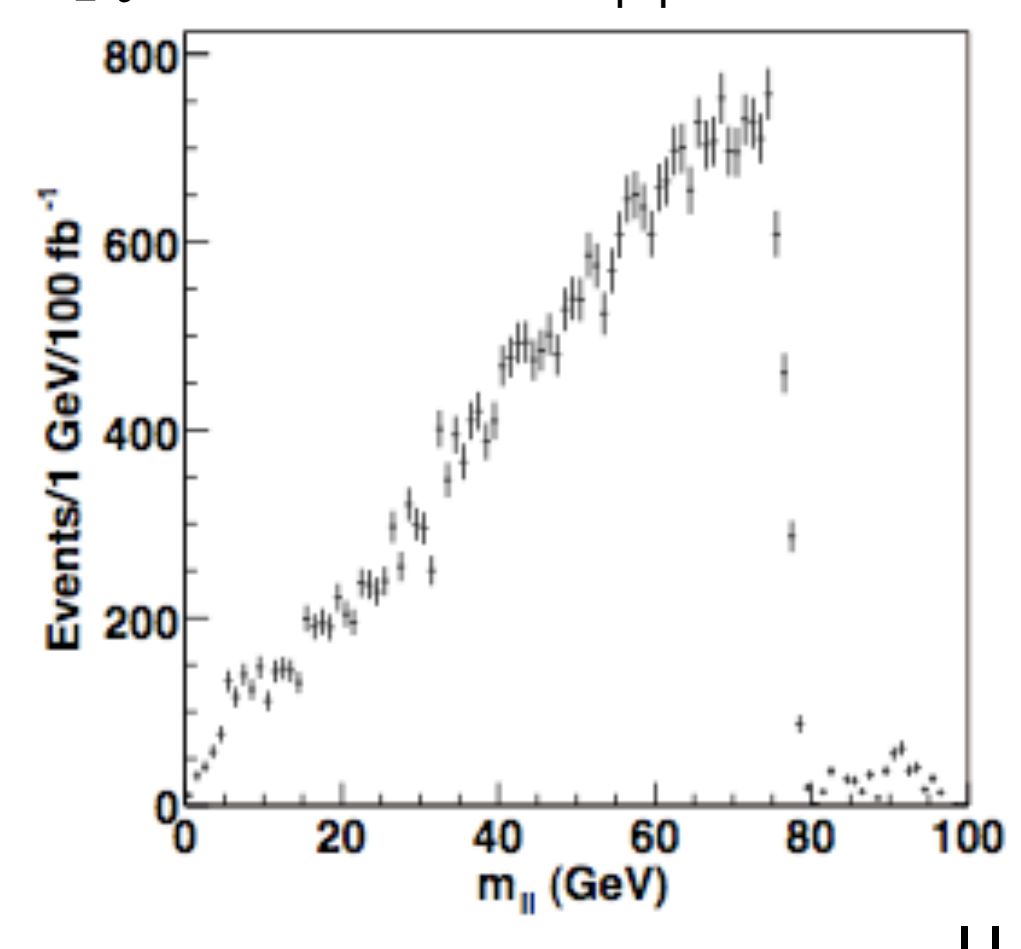




\section{Endpoint measurement}

\section{Model independence of endpoint}

\section{method}

* use same fit function and calibration for coannihilation point (SUI):

* lower cross section (factor 0.4)

* far tau very soft, hard to reconstruct

$\rightarrow$ different event selection:

* $\geq 2 \tau$ (track-seeded reconstruction)

米 2 jets: $\mathrm{PT}>100 / 50 \mathrm{GeV}$

* $\mathrm{E}_{\mathrm{T}}^{\text {miss }}>100 \mathrm{GeV}$

* elliptical cut in $\left(\mathrm{E}_{\mathrm{T}^{\mathrm{miss}}}, \mathrm{PT}^{\mathrm{jet} \mathrm{l}}+\mathrm{P}_{\mathrm{P}} \mathrm{T}^{\mathrm{jet} 2}\right)$ plane (semi-axes $450 \mathrm{GeV}$ ( $E_{T^{m i s s}}$, 500GeV (sum jet $\mathrm{PT}_{\mathrm{T}}$ ))
CERN-OPEN-2008-020

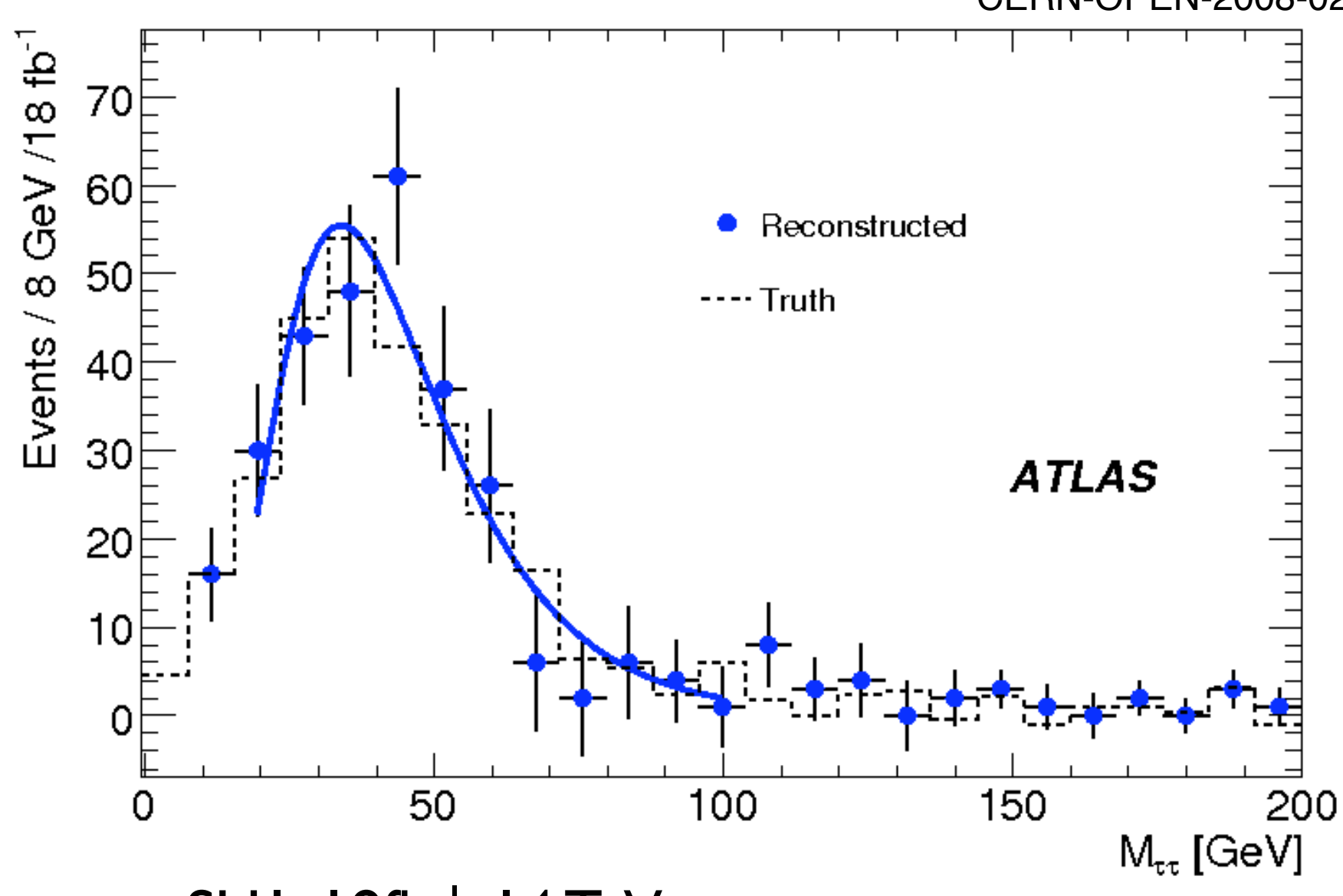

SUI, I8fb-1, I $4 \mathrm{TeV}$

$\rightarrow$ measured endpoint: (nominal value: $78 \mathrm{GeV}$ )

$\mathrm{m}_{\tau \tau}^{\max }=70 \pm 6.5^{\text {stat }} \pm 5^{\text {syst }} \mathrm{GeV}\left(18 \mathrm{fb}^{-1}\right)$

* ongoing work: use method in non-mSUGRA scenario 


\section{Polarization dependence}

\section{Influence of $\tau$ polarization on $\tau \tau$ mass spectrum:}
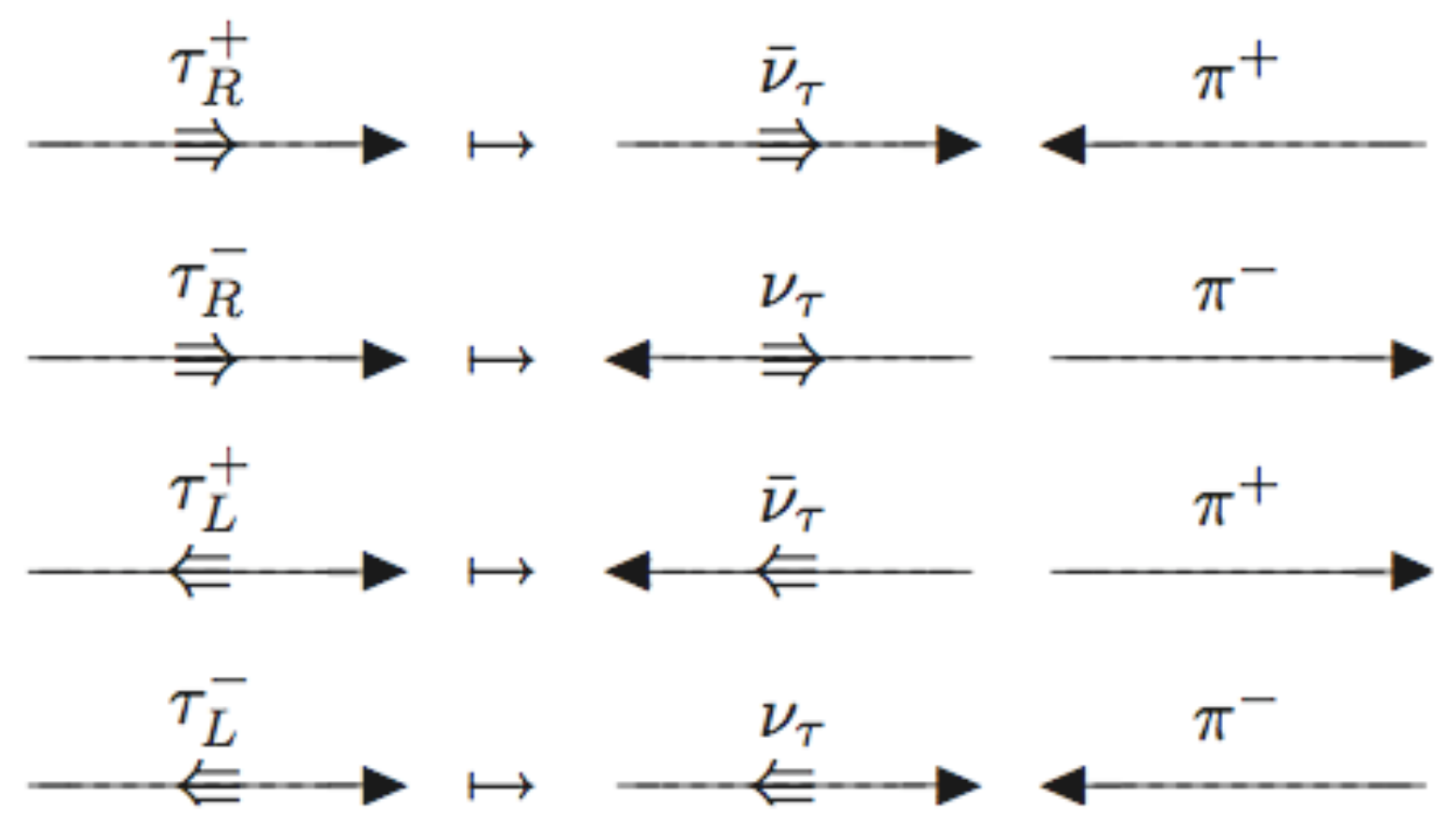

$\mathbf{T} \rightarrow \pi^{ \pm} \boldsymbol{V}_{\mathbf{T}}$ : fixed neutrino handedness

$\rightarrow$ pion momentum boosted (anti)parallel to tau momentum, dependent on tau polarization

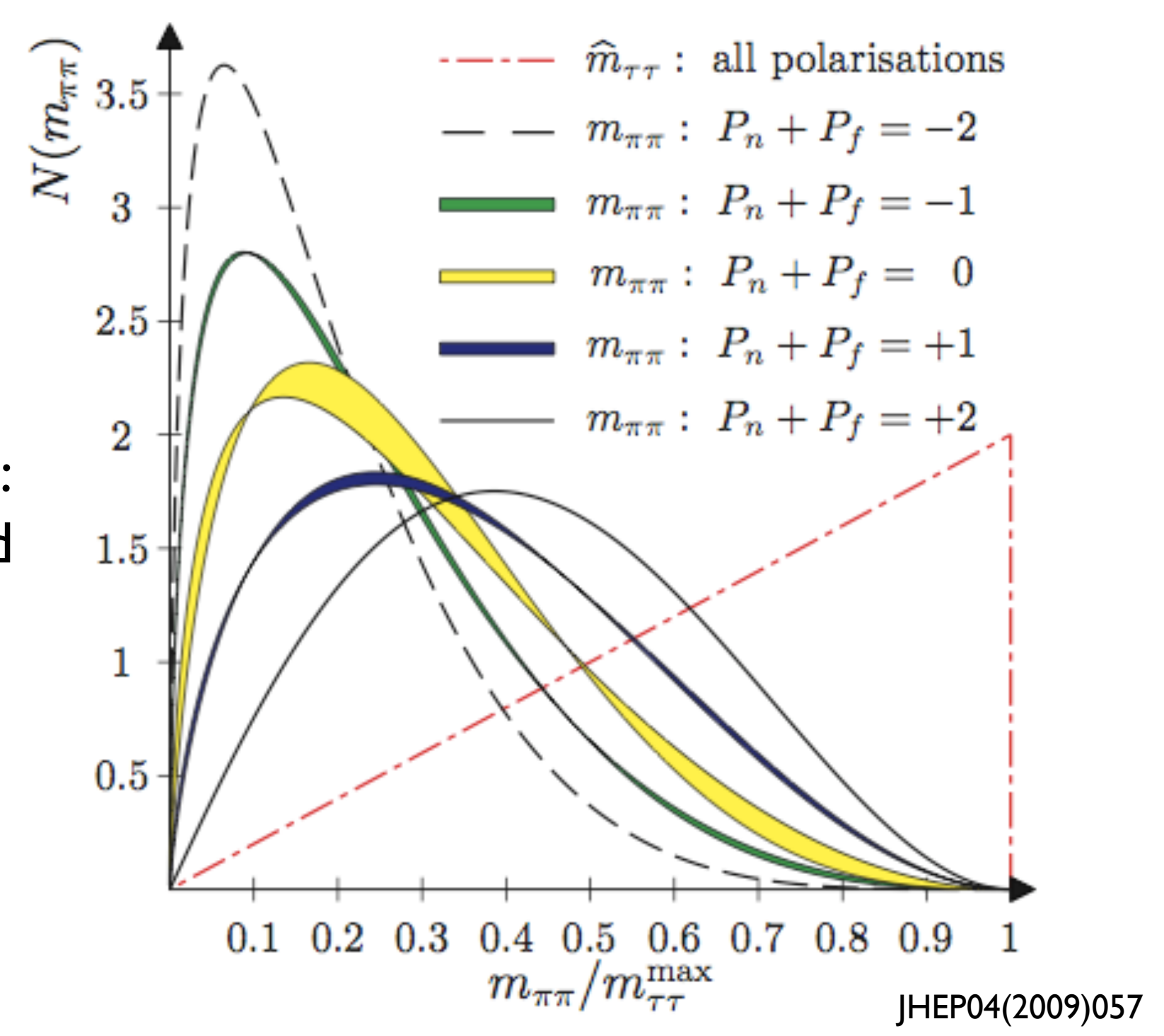

$\rightarrow$ mass spectra shifted for different $\tau$ polarizations:

* $\tau \tau$ spectrum depends on combination of near and far $\tau$ polarization $P_{n}+P_{f}$ and $P_{n} * P_{f}$

* Sum $P_{n}+P_{f}$ has far more impact than product

* Product $\mathrm{P}_{\mathrm{n}} * \mathrm{P}_{\mathrm{f}} \rightarrow$ variation bands 


\section{Endpoint and polarization measurement}

SUSY masses and $\tau$ polarizations change spectrum in different way

$\rightarrow$ fit spectrum with gaussian: more stable to polarization effects than log-normal function
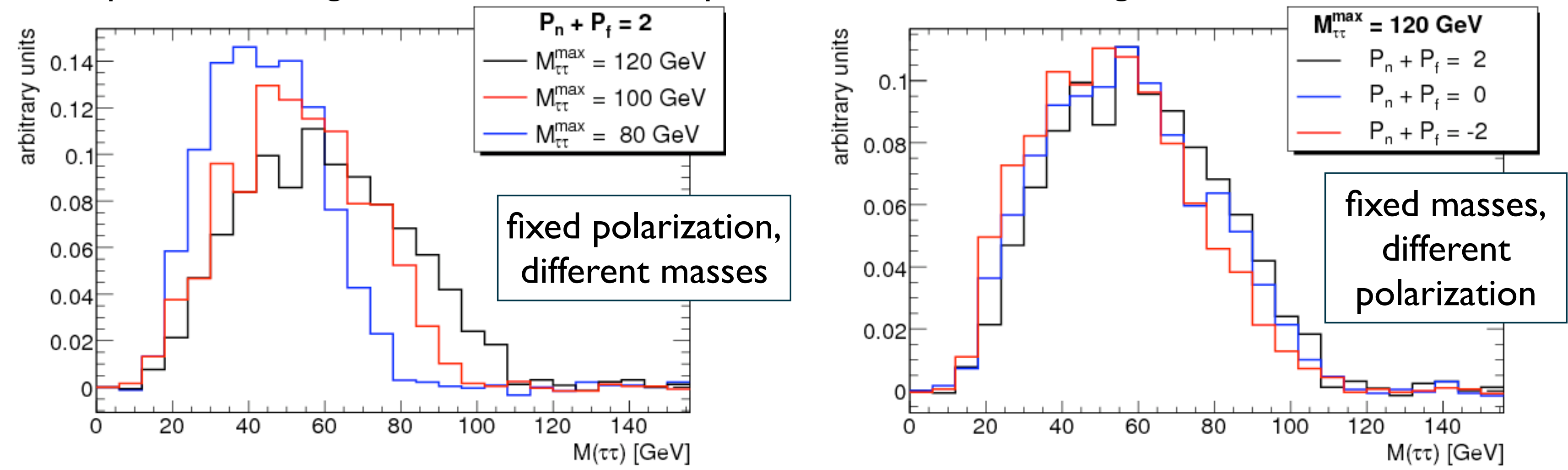

$\rightarrow$ measure both with 2 observables: $x\left(f^{\max }\right), x\left(0.1 *_{f \max }\right)$

$\rightarrow$ 2dim calibration

Fast detector

simulation

C. Zendler

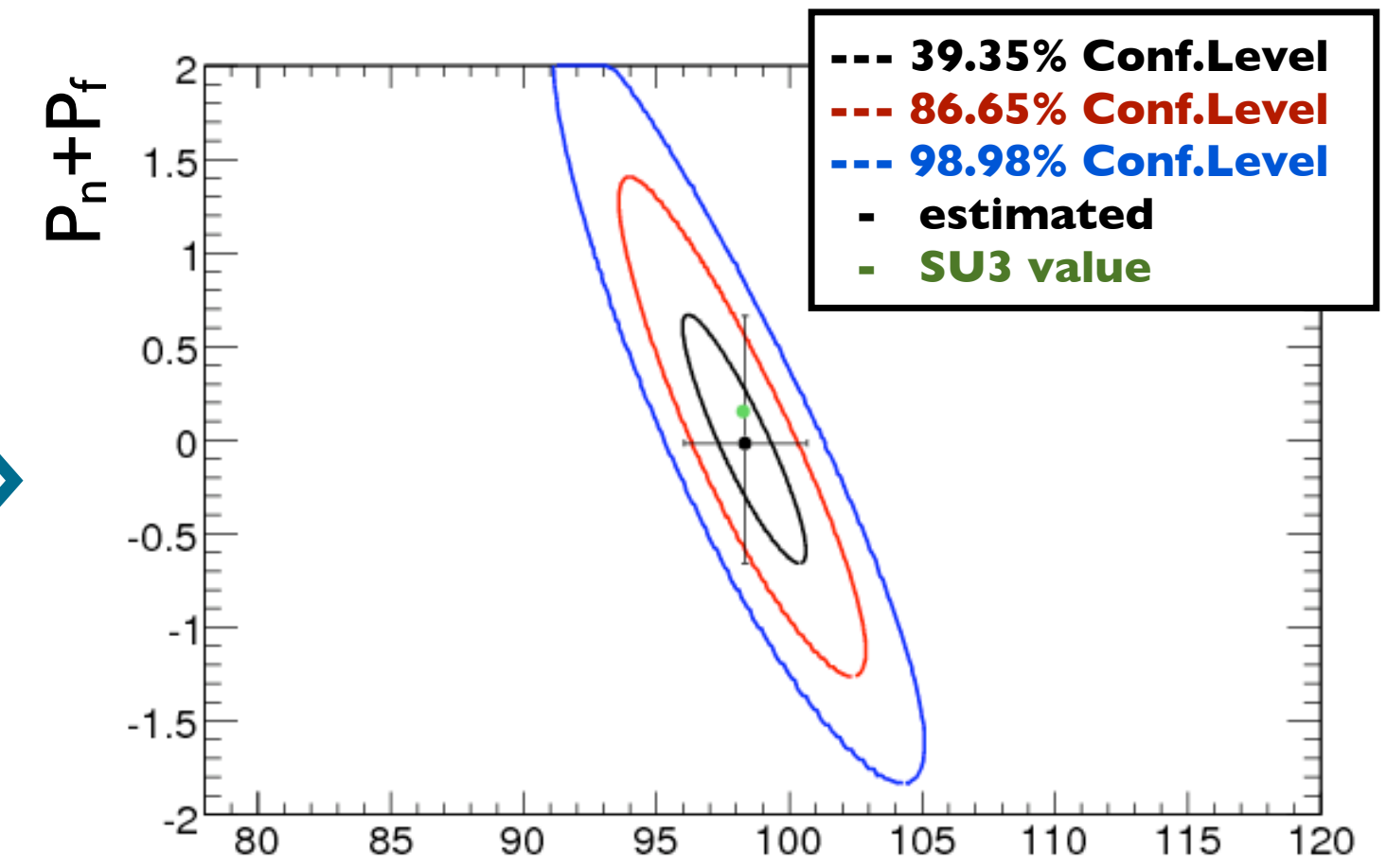

SUSY09, 05.-10..06.2009

endpoint [GeV] 


\section{Implications for SUSY parameters}

\section{Constraints on mixing angle and stau mass:}

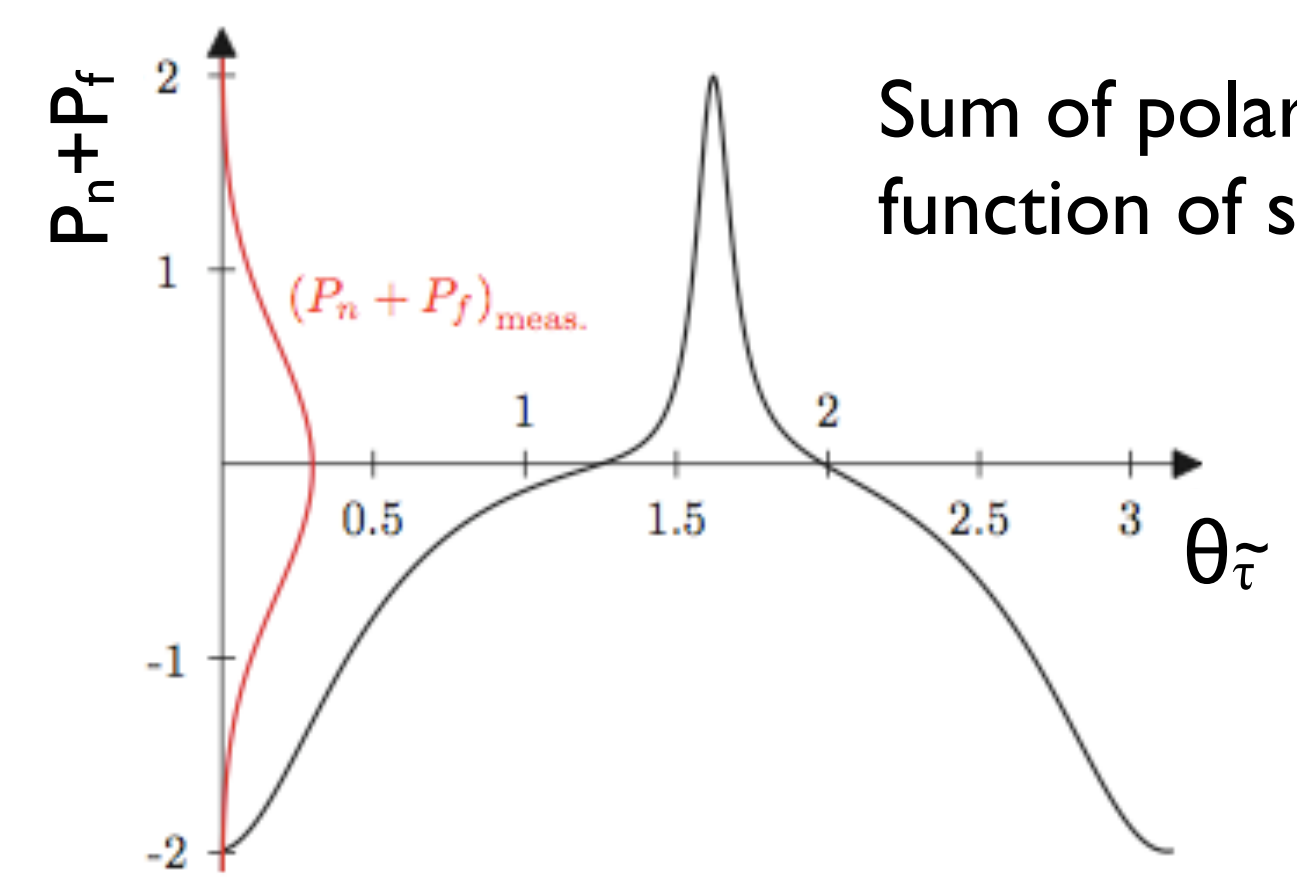

\section{assumption:} neutralino sector known from other measurements here: nominal values of SUI

Implication of measured values on SUSY parameters

JHEP04(2009)057
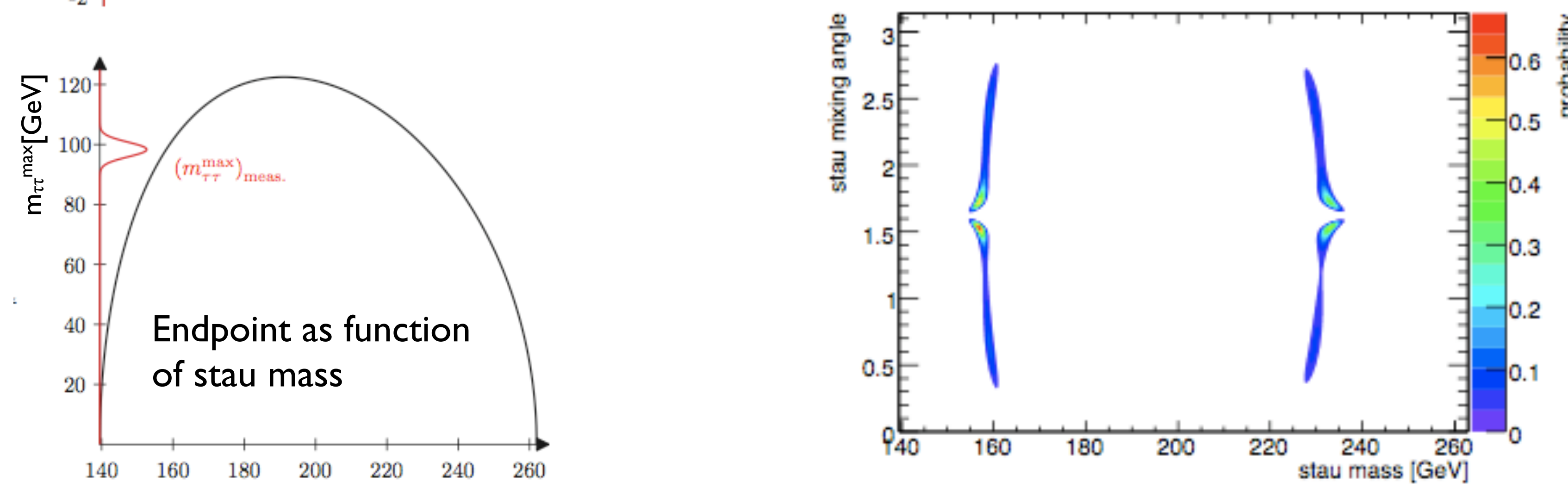

SUSY09, 05.-10..06.2009 


\section{Improvement by separation of decay modes}

\section{Possible improvement by separation of $\tau$ decay modes:}

* $\tau$ decay via vector mesons $\rho, a_{1}$ : direction of boost different for longitudinal/tranversal mesons:

米 longitudinally polarized: same as pion

米 transversally polarized meson: opposite behaviour

$\rightarrow$ overall effect depends on relative branching ratio

$$
\frac{1}{\Gamma_{v}} \cdot \frac{\mathrm{d} \Gamma_{v}}{\mathrm{~d} \cos \vartheta}=\underbrace{\left(\frac{m_{v}^{2}}{m_{\tau}^{2}+2 m_{v}^{2}}\left(1-P_{\tau} \cos \vartheta\right)\right)}_{\text {transversal }}+\underbrace{\left(\frac{\frac{1}{2} m_{\tau}^{2}}{m_{\tau}^{2}+2 m_{v}^{2}}\left(1+P_{\tau} \cos \vartheta\right)\right)}_{\text {longitudinal }}
$$

*⿻ $\mathrm{a}_{1}$ : same amount of longitudinal and transverse states

$\rightarrow$ inv. mass spectrum not affect by polarization effects

* $\rho$ : more longitudinal than transverse states

$\rightarrow$ inv. mass spectrum shifted in same direction as for $\pi$ decays

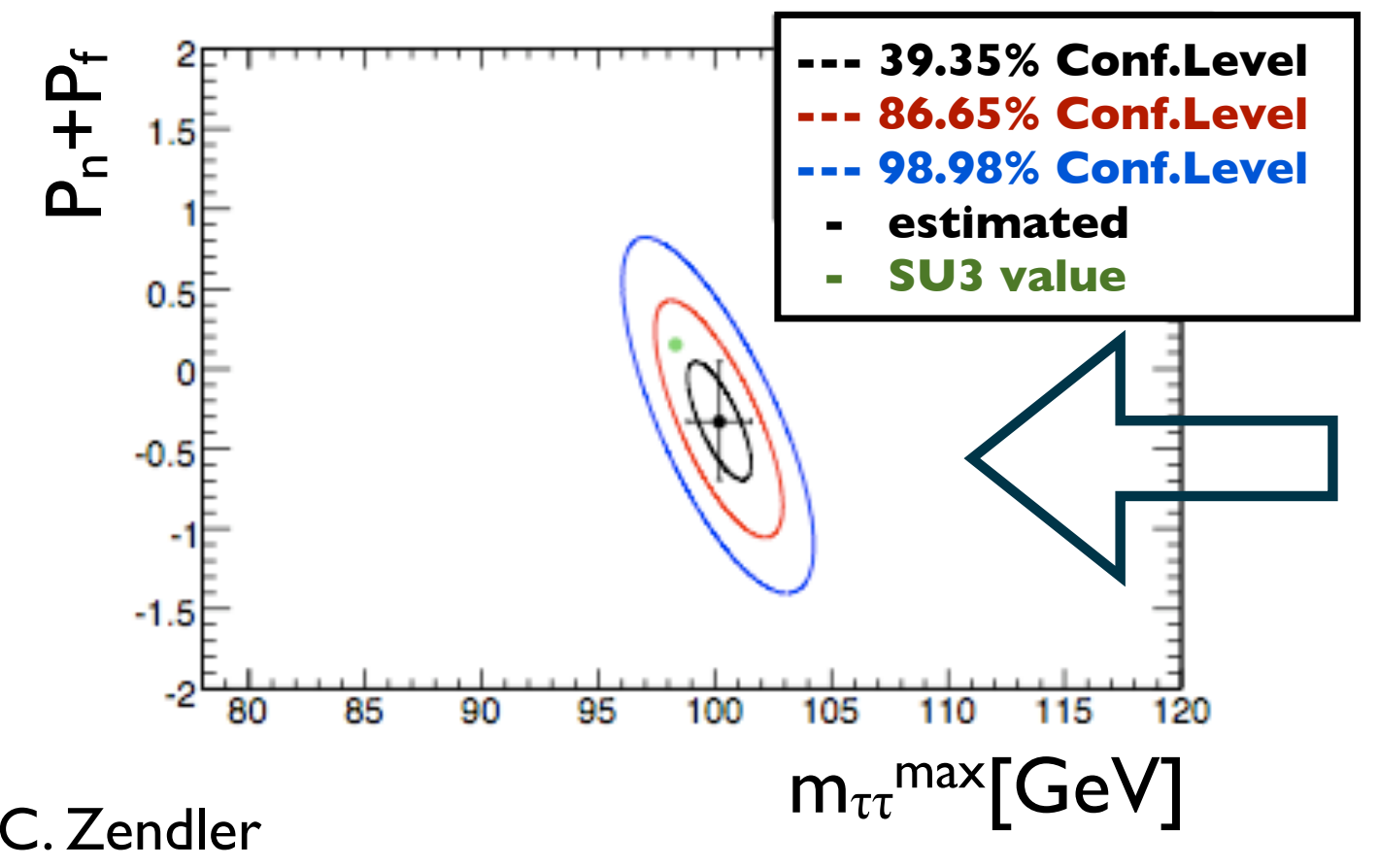

粉 divide spectrum in affected $(\pi, \mathrm{K}, \rho) /$ not affected

$\rightarrow 3 \times 2$ observables

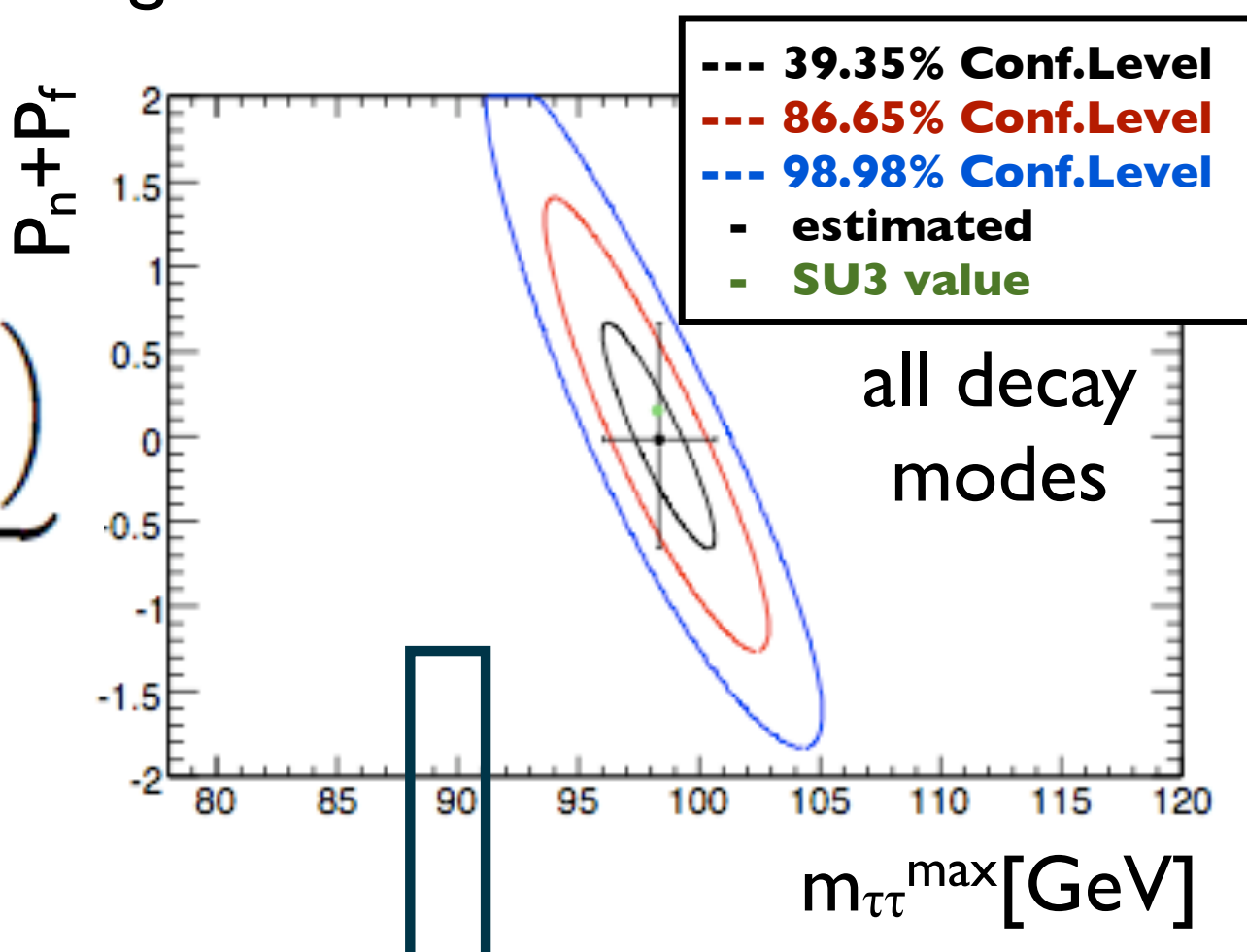

$39.35 \%$ Conf.Leve 3 value decay modes

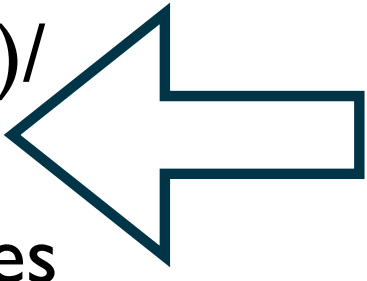

* Assume ideal tau decay mode separation

* Fake taus: assigned to decay mode with probability of BR

Fast detector SUSY09, 05.-10..06.2009 


\section{Tau decay mode reconstruction}

\section{Reconstruction of $\pi^{0}$}

\section{subclusters:}

* High granularity of ATLAS electromagnetic calorimeter allows reconstruction of isolated subclusters from $\pi^{0}$

I prong candidates:

\begin{tabular}{|l|c|c|c|}
\hline decay mode & $\begin{array}{c}\text { no } \pi^{0} \\
\text { subclusters }\end{array}$ & $\begin{array}{c}\mathrm{I} \pi^{0} \\
\text { subcluster }\end{array}$ & $\begin{array}{c}\geq 2 \pi^{0} \\
\text { subclusters }\end{array}$ \\
\hline$\tau \rightarrow \pi v_{\top}$ & $65 \%$ & $20 \%$ & $15 \%$ \\
\hline$\tau \rightarrow \rho v_{\top}$ & $15 \%$ & $50 \%$ & $35 \%$ \\
\hline$\tau \rightarrow \mathrm{a}_{1}\left(\rightarrow 2 \pi^{0} \pi\right) v_{\tau}$ & $9 \%$ & $34 \%$ & $57 \%$ \\
\hline
\end{tabular}

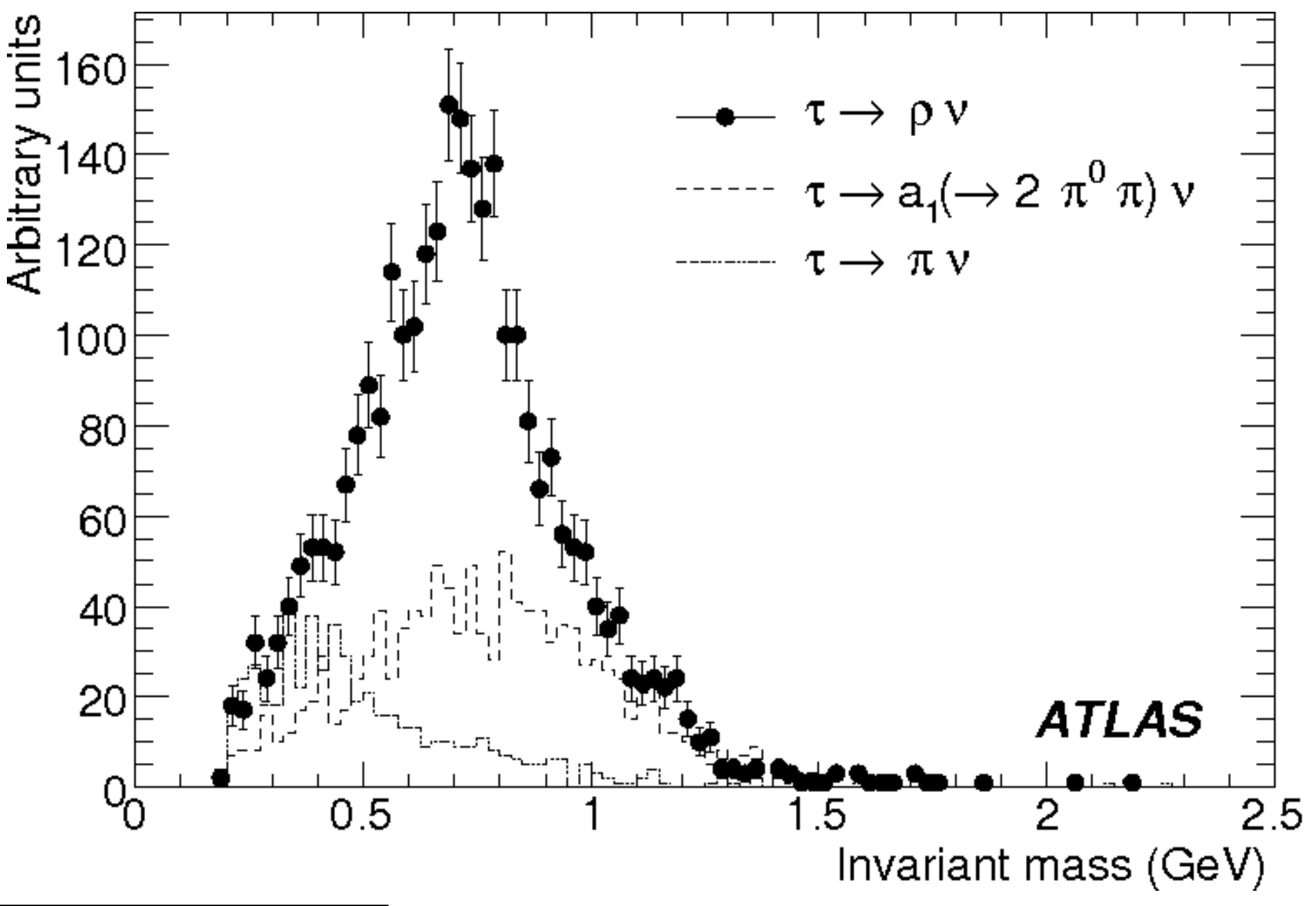

Invariant mass: candidates with at least one reconstructed $\pi^{0}$ subcluster, from $W \rightarrow \tau \nu_{T}$ 


\section{Summary}

* Taus are important for SUSY

$\rightarrow$ needed for searches and measurements

* Endpoint of $\tau \tau$ invariant mass spectrum can be measured accurately with $\sim \mathrm{fb}^{-1}$

$\rightarrow$ constraint on $\widetilde{\tau}_{1}$ mass

* Sum of polarizations can be measured additionally with $\sim 35 \mathrm{fb}^{-1}$

$\rightarrow$ constraint on $\tilde{\tau}$ mixing angle $\theta_{\tilde{\tau}}$

* Performance of tau reconstruction and identification crucial

$\rightarrow$ high reconstruction efficiency for visible signals

$\rightarrow$ high purity for meaningful signals

$\rightarrow$ information about tau decay could improve measurements significantly 


\section{backup}




\section{Tau reconstruction}

\section{Reconstruction}

* Begin with track based algorithm

* Seed: high quality track ( $\mathrm{PT}>6 \mathrm{GeV}$, requirements on \#hits in subdetectors and $\chi^{2} / \mathrm{ndf}$ )

* additional quality tracks ( $\mathrm{PT}>\mathrm{IGeV}$ ) in cone $\Delta \mathrm{R}<0.2$

* $\eta, \phi$ reconstruction with $\mathrm{PT}$-weighting of tracks

* check charge consistency

* search matching calorimeter seed

** Jet "Cone4HITopoJet" ( $\left.\mathrm{E}_{\mathrm{T}}>\mathrm{I0GeV},|\mathrm{n}|<2.5\right)$ within $\Delta \mathrm{R}<0.2$

$\rightarrow$ no match: track-only candidate $(5 \%)$

* $\mathrm{E}_{\mathrm{T}}$ from EnergyFlow algorithm

$\rightarrow$ match: track+calorimeter seeded candidate $(70 \%)$

* $\mathrm{E}_{\mathrm{T}}$ from cells of calorimeter based algorithm

* remaining clusters: seeds for calorimeter-only candidates (25\%)

* $\eta, \phi$ reconstruction from cluster

* looser track quality selection ( $\mathrm{PT}>\mid \mathrm{GeV}$ ) 


\section{Tau reconstruction and identification}

\section{Identification}

Discriminating variables:

米 variance $W_{\text {tracks }}$ (multiprong only)

* invariant mass of track system

* \#tracks in isolation cone

* electromagnetic radius $R_{\mathrm{em}}$

* \# $\eta$ strips with certain energy deposit

* width of the energy deposit

* $\mathrm{E}_{\mathrm{T}}$ fraction in cone $0.1<\Delta \mathrm{R}<0.2$ w.r.t. total energy in cone 0.2

* transverse energy at EM scale in core cone and isolation cone $E_{T}{ }^{\text {core }}, E_{T}{ }^{\text {isol }}, E_{T}{ }^{\text {isol }}$ AD

* hadronical $\mathrm{E}_{\mathrm{T}}$ fraction in core region w.r.t. sum PT of tracks

* visible mass

档 transverse impact parameter

* transverse flight path

档 $\pi^{0}$ subclusters safe variables:

Calorimter-based:

* radius in EM calorimeter

* isolation fraction

* width in strip layer

* $\mathrm{E}_{\mathrm{T}}(\mathrm{EM}) / \mathrm{E}_{\mathrm{T}}$

$\rightarrow$ uses only calorimeter information

additional for track+calorimterbased:

* width of track momenta

* $\mathrm{E}_{\mathrm{T}} / \mathrm{PT}$ of leading track

* electromagnetic and hadronic $E_{T}$ fraction w.r.t. sum PT of tracks

䊑 sum PT of tracks / $E_{T}$ 


\section{SUSY analyses with tau leptons}

mSUGRA benchmark points used:

\begin{tabular}{|c|c|c|c|c|c|}
\hline & $\begin{array}{c}\mathrm{m}_{0} \\
{[\mathrm{GeV}]}\end{array}$ & $\begin{array}{c}\mathrm{m}_{\mathrm{I} / 2} \\
{[\mathrm{GeV}]}\end{array}$ & $\begin{array}{c}\mathrm{A}_{0} \\
{[\mathrm{GeV}]}\end{array}$ & $\tan \beta$ & $\operatorname{sgn} \mu$ \\
\hline $\begin{array}{c}\text { SUI } \\
\text { coannihilation }\end{array}$ & 70 & 350 & 0 & 10 & + \\
\hline $\begin{array}{c}\text { SU3 } \\
\text { bulk }\end{array}$ & 100 & 300 & -300 & 6 & + \\
\hline $\begin{array}{c}\text { SU6 } \\
\text { funnel }\end{array}$ & 320 & 375 & 0 & 50 & + \\
\hline
\end{tabular}

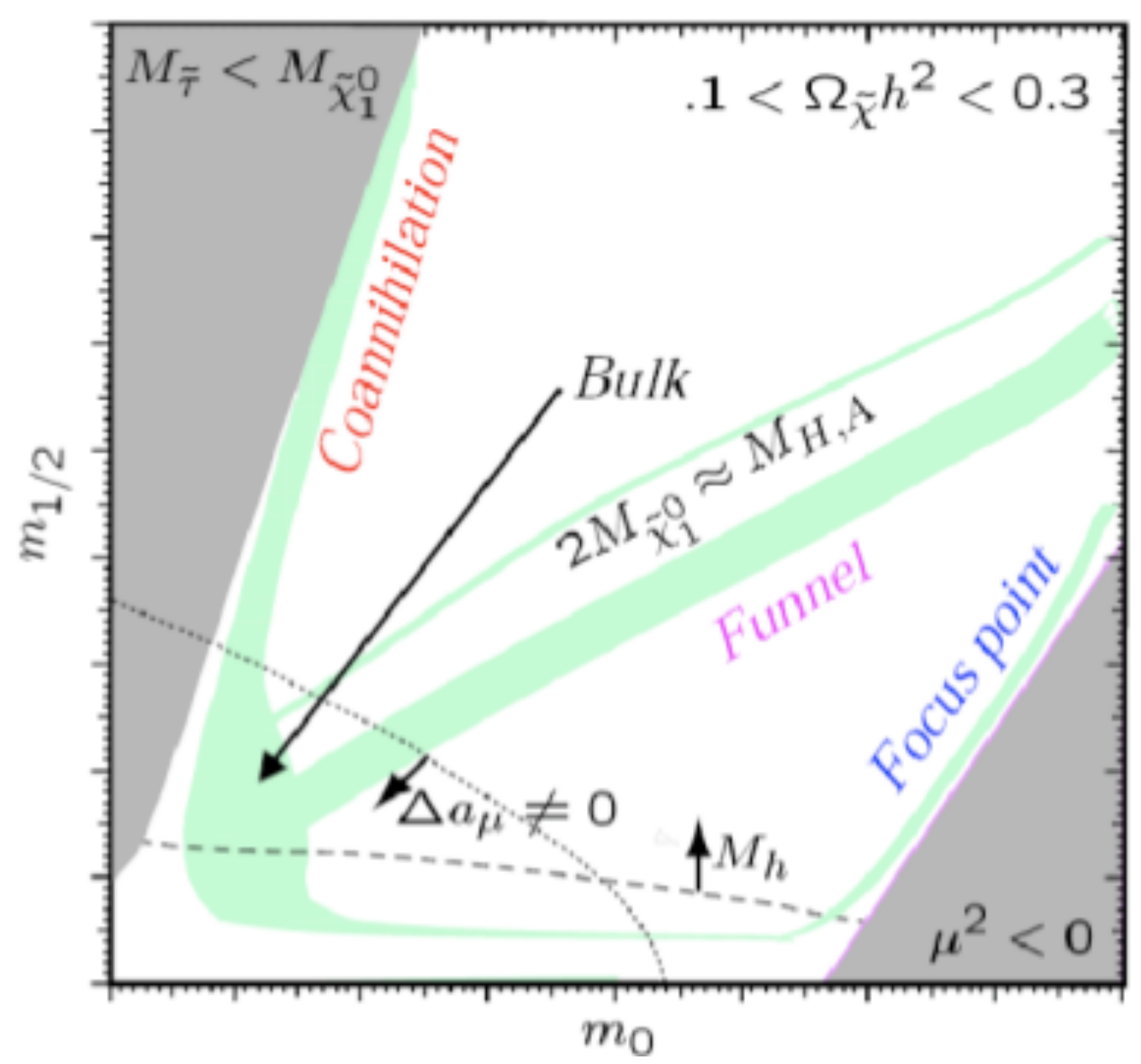




\section{Tau polarization}

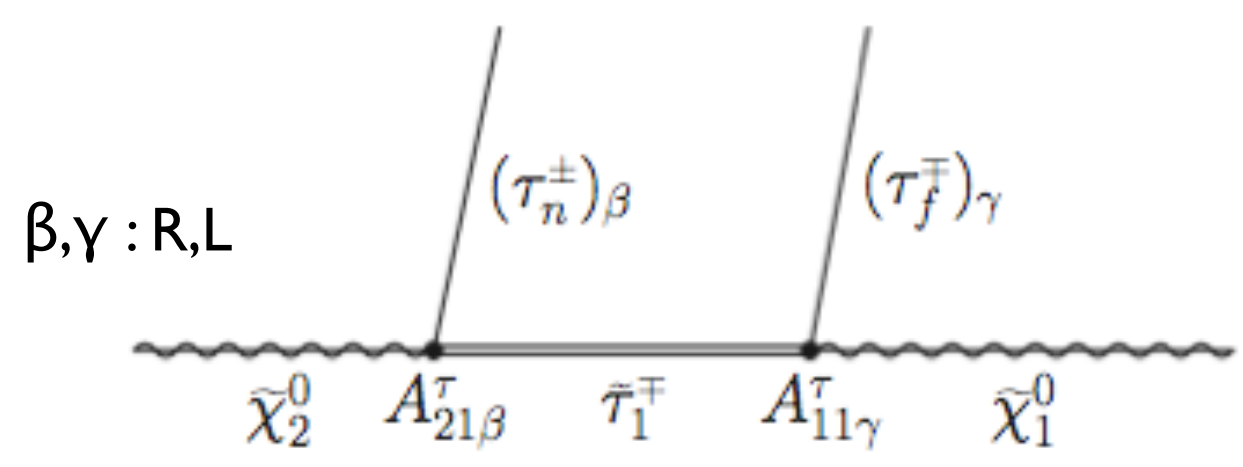

$$
\begin{aligned}
A_{j 1 L}^{\tau} & =-\frac{m_{\tau}}{\sqrt{2} m_{W} \cos \beta} N_{j 3}^{*} \sin \theta_{\tilde{\tau}}+\frac{1}{\sqrt{2}}\left(N_{j 2}^{*}+N_{j 1}^{*} \tan \theta_{W}\right) \cos \theta_{\tilde{\tau}} \\
A_{j 1 R}^{\tau} & =-\frac{m_{\tau}}{\sqrt{2} m_{W} \cos \beta} N_{j 3} \cos \theta_{\tilde{\tau}}-\sqrt{2} N_{j 1} \tan \theta_{W} \sin \theta_{\tilde{\tau}},
\end{aligned}
$$

$\rightarrow$ polarization: $\quad P=\frac{\left(A_{j 1 R}^{\tau}\right)^{2}-\left(A_{j 1 L}^{\tau}\right)^{2}}{\left(A_{j 1 R}^{\tau}\right)^{2}+\left(A_{j 1 L}^{\tau}\right)^{2}}$,

Ditau mass spectrum for $\mathbf{T} \rightarrow \boldsymbol{\pi}^{ \pm} \mathbf{V}_{\mathbf{T}}$

$$
\begin{aligned}
& N\left(m_{\pi \pi}\right)=4 m\left\{\left(P_{n} \cdot P_{f}\right)[\right.\left.\ln m\left(\ln m+4 m^{2}+4\right)+4\left(1-m^{2}\right)\right]+ \\
&\left.+\left(P_{n}+P_{f}\right)\left[m^{2}-2 \ln m-1-\ln ^{2} m\right]+\ln ^{2} m\right\}
\end{aligned}
$$




\section{Endpoint and polarization measurement}

SUSY masses and $\tau$ polarizations change spectrum in different way

$\rightarrow$ fit spectrum with gaussian: more stable to polarization effects that log-normal function

$\rightarrow$ possible to measure both with 2 ovservables: $x\left(f^{\max }\right), \times\left(0.1 *^{m} \max \right)$

$\rightarrow 2 \operatorname{dim}$ calibration needed:
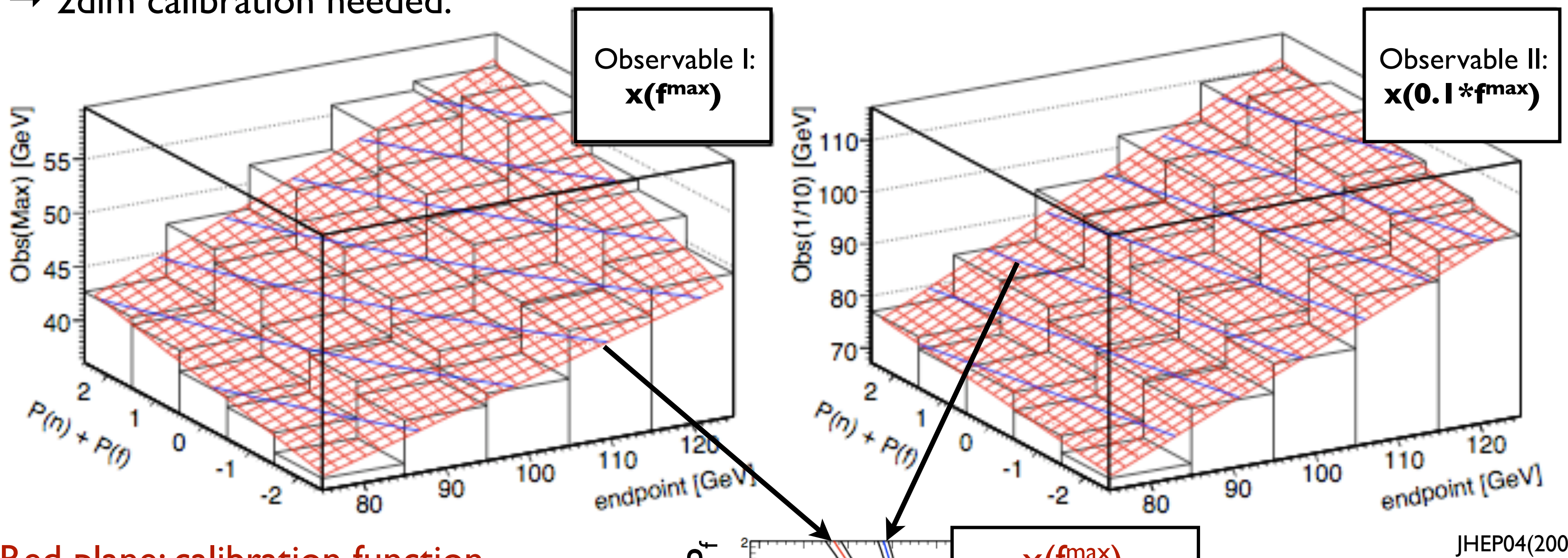

Red plane: calibration function Blue lines: equipotential lines

Fast detector simulation

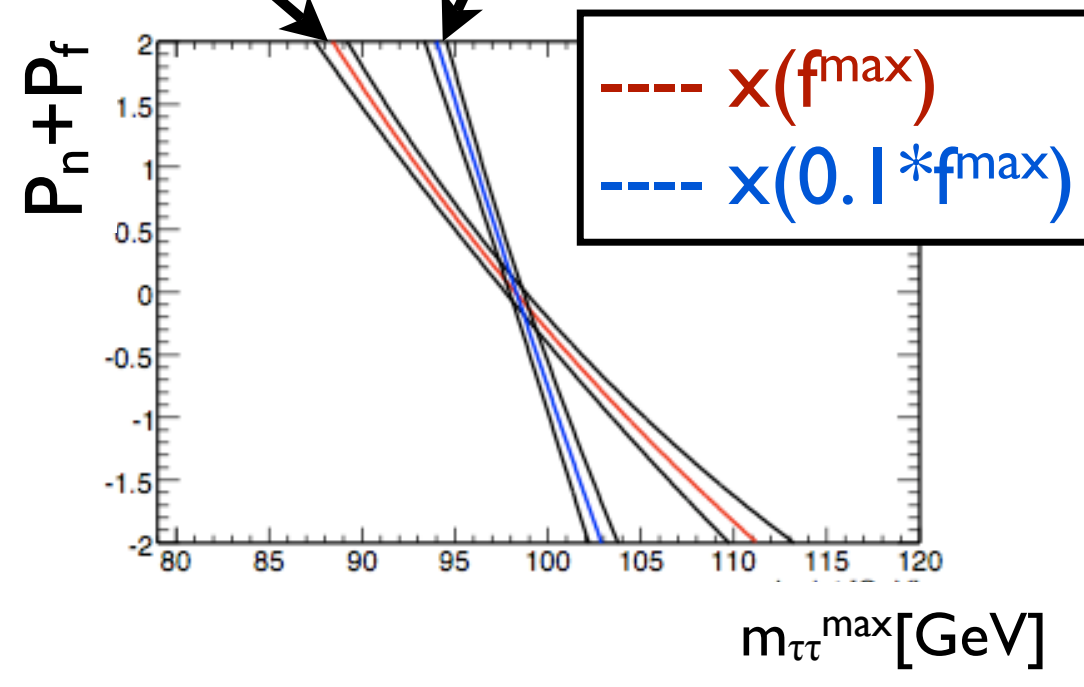

